Schmerz 2010 - 24:9-11

DOI 10.1007/s00482-009-0862-x

Online publiziert: 22. Januar 2010

(c) Deutsche Gesellschaft zum Studium

des Schmerzes. Published by Springer

Medizin Verlag - all rights reserved 2010

\section{B. Zernikow $\cdot$ T. Hechler $\cdot$ S. Schroeder}

Vodafone Stiftungsinstitut und Lehrstuhl für Kinderschmerztherapie und Pädiatrische Palliativmedizin, Vestische Kinder- und Jugendklinik Datteln, Universität Witten/Herdecke, Datteln

\title{
Neues Design des Deutschen Schmerzfragebogens für Kinder und Jugendliche (DSF-KJ)
}

Die Erfassung chronischer Schmerzen im Kindes- und Jugendalter kann nur durch eine multidimensionale Erfassung des Schmerzgeschehens erfolgen. Während im Erwachsenenbereich der Deutsche Schmerzfragebogen standardmäßig eingesetzt wird [1], fehlt es an vergleichbaren Anamneseschemata im Kindes- und Jugendbereich. Der Deutsche Schmerzfragebogen für Kinder und Jugendliche (DSFKJ; [3]), dessen Entwicklung und psychometrische Überprüfung in diesem Heft ausführlich präsentiert wird, schließt die Lücke in der Diagnostik für Kinder und Jugendliche.

Auf der Grundlage der Erkenntnisse der Ergebnisse von Schroeder et al. [3] wurde der DSF-KJ in Expertengremien kontinuierlich weiterentwickelt. Die Weiterentwicklungen umfassen dabei

1. Veränderungen des Designs, um das Ausfüllen dieses Fragebogens zu erleichtern,

2. inhaltliche Änderungen, z. B. die themenspezifische Aufteilung innerhalb des DSF-KJ nach 6 Kernthemen,

3. das Hinzufügen neuer Items, wie beispielsweise die detaillierte Erfassung des Migrationsstatus, und

4. die Überarbeitung einzelner Items, für die die Analysen Verständnisschwierigkeiten (im Sinne fehlender Items) aufzeigten [3].

Eine detaillierte Auflistung der Veränderungen finden Sie in $\bullet$ Infobox 1. Der Fragebogen kann gegen die Druck- und Portokosten bei uns bezogen werden (http://www.vodafone-stiftungsinstitut. de). Für die Verwendung der im Fragebogen enthaltenen und urheberrechtlich geschützten Schmerzempfindungsskala (SES) müssen Gebühren an den HogrefeVerlag abgeführt werden.

Der DSF-KJ trägt zur Optimierung der Diagnostik chronischer Schmerzen im Kindes- und Jugendalter bei. Zudem bietet er eine gute Grundlage, die diagnostischen Empfehlungen der Pediatric Initiative on Methods, Measurement, and Pain Assessment in Clinical Trials (PedIMMPACT; [2]) auch im deutschen Sprachraum umzusetzen. Geplant ist dazu, den DSF-KJ im Rahmen multizentrischer Studien weiter hinsichtlich seiner psychometrischen Charakteristika zu überprüfen.

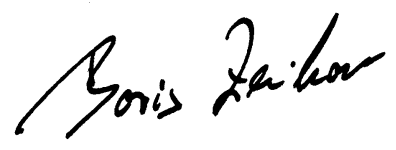

B. Zernikow

\section{Korrespondenzadresse}

Prof. Dr. B. Zernikow

Vodafone Stiftungsinstitut und Lehrstuhl für Kinderschmerztherapie und Pädiatrische Palliativmedizin, Vestische Kinder- und Jugendklinik Datteln, Universität Witten/Herdecke Dr.-Friedrich-Steiner Str. 5, 45711 Datteln B.Zernikow@kinderklinik-datteln.de

Interessenkonflikt. Keine Angaben.

\section{Literatur}

1. Deutsche Gesellschaft für Schmerztherapie e.V, Deutsche Gesellschaft zum Studium des Schmerzes (2007) Deutscher Schmerzfragebogen. http://www.deutscher-schmerzfragebogen.de
2. McGrath PJ, Walco GA, Turk DC et al (2008) Core outcome domains and measures for pediatric acute and chronic/recurrent pain clinical trials: PedIMMPACT recommendations. J Pain 9: 771-783

3. Schroeder S, Hechler T, Denecke H et al (2009) Deutscher Schmerzfragebogen für Kinder, Jugendliche und deren Eltern (DSF-KJ) - Ein multimodaler Fragebogen zur Diagnostik und Therapie chronischer Schmerzen im Kindes- und Jugendalter. Schmerz 24: 23-37 


\section{Weiterentwicklungen des DSF-KJ auf der Grundlage der Ergebnisse von Schroeder et al. [3]}

\section{1) Veränderungen des Designs}

\section{- Farbleitschema:}

- Kindern (grün), Jugendlichen (orange) und Eltern (lila) ist jeweils eine Farbe fest zugeordnet.

- Durch Abstufung der Farbtiefe auf dem Cover sind verschiedene Bögen (Erstbögen und Verlauf) leicht voneinander zu unterscheiden.

- Cover:

- Ziel war ein Design, das einerseits einheitlich für alle Altersgruppen ist, aber sowohl Kinder wie Jugendliche als auch Eltern anzusprechen vermag. Außerdem sollte es den Begriff "Schmerz" nicht negativ interpretieren, sondern neutral. Daher kam z. B. kein Blitz oder Schwert infrage.

- Um alle Altersgruppen anzusprechen, fiel die Wahl auf eine (eher abstrakte) Grafik und nicht auf eine kindliche Zeichnung. Eine kleine, informelle Befragung von kindlichen und jugendlichen stationären Schmerzpatienten ergab, dass diese ein grafisches Design bevorzugen anstatt einer Kinderzeichnung (Beispiel: Migränetagebuch).

- Die dargestellte Linie repräsentiert die Multidimensionalität von Schmerz bzw. des multidimensional aufgebauten Fragebogens. Abgeleitet wurde sie aus einer Z-Linie einer QST-Messung (Schmerzprofil). Darüber hinaus lässt die Linie aber auch Raum für die Interpretation eines (chronischen) Schmerzverlaufs (mit Höhen und Tiefen, Fortschritten und Rückschlägen).

- Innenteil:

- Durch die Farbgestaltung und wechselweise Unterlegung mit und ohne Hintergrundfarbe soll der Bogen optisch aufgelockert werden, sodass er nicht zu lang wirkt, sondern sich auf viele kleine Einheiten verteilt und Abwechslung bringt. Ziel ist, dass Patienten den Bogen leichter ausfüllen können.

- Kleine weiße Dreiecke am linken Seitenrand heben zusätzlich ausgewählte Fragen hervor, um die Aufmerksamkeit zu erhalten.

- Die Gliederung erfolgt klar in aufeinander aufbauenden Kapiteln. Fragen gehören thematisch zusammen.

- Platz für Notizen gibt es in der Regel immer auf den hinteren Seiten. Es können aber auch wie bisher Vorder- und Rückseite sowie die 1. Seite (Impressum) beschrieben werden, da das Design genügend Freiraum lässt.

\section{2) Inhaltliche Änderungen}

\section{Allgemein:}

Klarere Gliederung in Kapitel und Abschnitte. Im alten Fragebogen waren die Fragen zum Teil verstreut (passend zum Seitenumbruch) und die Reihenfolge in den verschiedenen Versionen [ $\mathrm{E}$ (Eltern) - K (Kind) - J (Jugendlicher) bzw. Erstkontakt - Verlauf] untereinander verschieden. Dies wurde komplett über alle Versionen hinweg vereinheitlicht. Über- und Unterschriften strukturieren nun den Bogen.

Folgende Kapitel gliedern den Erstbogen:

1. Soziodemografische Anamnese und Familienanamnese

2. Schmerzcharakteristika

3. Medizinische Vorgeschichte

4. Auslösende und schmerzbeeinflussende Faktoren

5. Schmerzbewältigung

6. Schmerzbezogene Beeinträchtigung

Folgende Kapitel gliedern die Verlaufsbögen:

1. Schmerzcharakteristik

2. Schmerzbewältigung

3. Schmerzbezogene Beeinträchtigung

4. Schmerztherapie

Der Verlaufsbogen wurde getrennt in Versionen für Kinder, Erwachsene und Jugendliche, um ein paralleles und unabhängiges Ausfüllen zu gewährleisten.

Alle Bögen wurden auf sprachliche Fehler hin überprüft sowie darauf, dass in allen Bögen jeweils identische Formulierungen auftauchen.

\section{3) Hinzufügen neuer Items}

- Fragen zum Migrationsstatus in der Eltern- und Jugendlichenversion, übernommen aus dem Kinder- und Jugendgesundheitssurvey (KiGGS). Diese liefern einen Algorithmus zur Bestimmung des Migrationsstatus.

- Die Abfrage von Schlafstörungen (Art und Dauer) wurde aufgenommen.

- Eine Frage zur Menarche und Menstruation wurde hinzugefügt, um menstruationsassoziierte Schmerzen zu erfassen (v. a. bei Migräne).

- Als neue Frage wurde formuliert, seit wann (falls ja) Dauerschmerzen bestehen.

- Im Verlaufsbogen wurde eine ergänzende Frage eine zur Zufriedenheit mit der Schmerztherapie (numerische Ratingskala 0-10) aufgenommen. 


\section{4) Überarbeitung einzelner Items/Löschung einzelner Items}

Schmerzintensitäten: Die Frage nach aktuellem Schmerz und schwächstem Schmerz wurde gestrichen. Erfragt werden damit nur noch maximaler und Durchschnittschmerz.

Überarbeitete Items:

- Begleitsymptome bei Eltern: Häufige Symptome zum Ankreuzen (statt wie bisher nur Freitext).

- Vorbehandler bei Eltern: Häufige Fachrichtungen zum Ankreuzen (statt wie bisher nur Freitext).

- Für die numerische Ratingskala (NRS 0-10) wurde die schriftliche Instruktion überarbeitet und präzisiert.

- Tabellen und deren Beispiele wurden überarbeitet, insbesondere bei:

- Abfrage der Art der Vorbehandlungen (präziser und umfassender, mehr Beispiele vorgegeben).

- Die medizinische Vorgeschichte des Patienten wird genauer erfasst. Erfragt werden alle stationären Behandlungen (schmerz- und anderweitig bedingt), da die Erfahrung zeigte, dass Eltern manche Aufenthalte nicht angaben, weil sie diese nicht auf Schmerzen bezogen haben. Erfragt werden zudem alle Medikamente der letzten 3 Monate (nicht nur Schmerzmedikamente), um aktuelle Erkrankungen und deren Behandlung zu identifizieren, sowie alle jemals eingesetzten Schmerzmedikamente.

- Fragen zum familiären und beruflichen Status wurden präzisiert und ergänzt.

- Die grafische Darstellung von Schmerzverläufen wurde optimiert, um ein besseres Verständnis zu erreichen.

- Abfrage der Schmerzhäufigkeit: Neue Kategorien erfassen nun exakter die Häufigkeit.

- Abfragezeiträume wurden angepasst bzw. erstmals präzisiert:

- Schmerzintensitäten: Erfragt werden bei Jugendlichen und Eltern die letzten 7 Tage sowie die letzten 4 Wochen, bei Kindern nur die letzten 7 Tage. Grund: Zur Erfassung chronischer Schmerzen, insbesondere der chronisch rezidivierenden Schmerzen, ist die Abfrage eines 4-Wochen-Intervalls klinisch sinnvoll. Ergänzend werden die letzten 7 Tage erfragt, um eine Vergleichbarkeit mit Daten aus klinischen Studien zu erhalten.

- Schulfehltage: Erfragt werden die letzten 3 Monate anstatt der letzten 4 Wochen. Dies entspricht dem Standard in der Literatur und verbessert die statistische Auswertbarkeit. Zusätzlich werden die letzten 4 Schulwochen erfragt, wenn es eine Änderung gibt, um den Anforderungen des MDK bei stationären Patienten Rechnung zu tragen.

- Medikamenteneinnahme: Fehlende Präzisierung des Zeitraums wurde ergänzt.

— Grafischer Schmerzverlauf: Fehlende Präzisierung des Zeitraums wurde ergänzt.

- Beeinträchtigung: Fehlende Präzisierung des Zeitraums wurde ergänzt.

- Anzahl der Arztbesuche: Abfrage der letzten 3 Monate. 